\title{
Application of Photonic Crystal Enhanced Fluorescence to Detection of Low Serum Concentrations of Human IgE Antibodies Specific for a Purified Cat Allergen (Fel d1)
}

\author{
Yafang $\operatorname{Tan}^{1}$, John F. Halsey ${ }^{2}$, Tiantian Tang ${ }^{1}$, Scott Vande Wetering ${ }^{3}$, \\ Elaine Taine ${ }^{3}$, Mark Van Cleve $^{3}$, Brian T. Cunningham ${ }^{1,4}$ * \\ ${ }^{1}$ Department of Electrical and Computer Engineering, University of Illinois at Urbana- \\ Champaign \\ ${ }^{2}$ Exalt Diagnostics, Urbana-Champaign, IL \\ ${ }^{3}$ Hycor Biomedical, Indianapolis, IN \\ ${ }^{3}$ Department of Bioengineering, University of Illinois at Urbana-Champaign \\ ${ }^{*}$ Corresponding author: 208 North Wright Street, Urbana, Illinois, 61801. \\ email: bcunning@illinois.edu, phone: 217-265-6291
}




\section{Abstract}

We demonstrate the detection of low concentrations of allergen-specific Immunoglobulin E ( $\lg E$ ) in human sera using a Photonic Crystal Enhanced Fluorescence (PCEF) microarray platform. The Photonic Crystal (PC) surface, designed to provide optical resonances for the excitation wavelength and emission wavelength of Cy5, was used to amplify the fluorescence signal intensity measured from a multiplexed allergen microarray. Surface-based sandwich immunoassays were used to detect and quantify specific IgE antibodies against a highly purified cat allergen (Fel d1). A comparison of the lowest detectable concentration of $\operatorname{lgE}$ measured by the PC microarray system and a commercially available clinical analyzer demonstrated that the PCEF microarray system provides higher sensitivity. The PCEF system was able to detect low concentrations of specific $\lg \mathrm{E}(\sim 0.02 \mathrm{kU} / \mathrm{L})$, which is 5 to 17 -fold more sensitive than the commercially available FDA-approved analyzers. In preliminary experiments using multi-allergen arrays, we demonstrate selective simultaneous detection of IgE antibodies to multiple allergens. 


\section{Introduction}

In industrial countries, more than $20 \%$ of the population suffers from type I allergies (i.e. Mediated by Immunoglobulin E), representing a major health problem in the western world (Conroy 2013; Wills-Karp et al. 2001; Zuberbier et al. 2014). The clinical evaluation of an allergic disorder typically involves use of the clinical history, physical examination and a test to confirm sensitization to the allergen. (Burks et al. 2011). Sensitization can be measured by skin testing with allergen extracts (Gergen et al. 1987; Hagy and Settipane 1971; Lieberman and Sicherer 2011) or blood tests (Feeney et al. 2012; Rudenko et al. 2013; Schellenberg and Adkinson 1975; Sicherer et al. 2012; Wahyuni et al. 2003). A skin test is done by monitoring a patient's reactions after a small amount of a suspected allergen is placed on or below the skin, while a blood test is an immunoassay that measures the concentration of Immunoglobulin E $(\lg E)$ against specific allergens in the patient's blood. Blood tests have several advantages over skin tests. First, they are more convenient as they involve only a standard blood draw, and are considered to be safer, since they are performed in vitro, and thus do not expose the patient to allergens (Howanitz 2005; Turkeltaub and Gergen 1989). Second, blood tests are performed as an alternative to skin tests for patients who have severe symptoms and cannot stop taking medication (Pipkorn et al. 1989). Moreover, studies have shown that the total amount of $\lg E$ against some allergens can predict the severity of symptoms (Sampson and Ho 1997; Yazdanbakhsh et al. 2002).

The currently available analysis systems for blood tests primarily rely upon crude allergen extracts prepared from various allergen-containing biological materials (e.g. pollens, foods, etc). These extracts contain a variety of allergenic and non-allergenic 
components and are often difficult to standardize with respect to their allergen content or potency. Therefore, extract-based diagnostics may not adequately discriminate between patients who are sensitized to different allergen components (Jutel et al. 2005; Valenta et al. 1999). For the same reason, it is difficult to provide accurate allergy therapy to individual patients if poorly defined allergen extracts are utilized as components of diagnostic assays. However, by applying current protein and DNA technology to the field of allergen identification, it is now possible to produce the major allergens for the most important allergens in a purified form (Harwanegg et al. 2003; Jahn-Schmid et al. 2003). Using these purified allergens, each individual patient's risk can be accurately assessed (Chapman et al. 1983; Kazemi-Shirazi et al. 2000; Östblom et al. 2008; Simpson et al.). The most useful treatment plan can be designed according to his/her sensitization profile. Since a variety of purified and recombinant allergens are now available, a comprehensive monitoring of the patient's $\lg E$ reactivity profile to a great number of different allergen molecules requires a new type of test that can provide multi-allergen detection.

In addition to the fact that the present commercial platforms fail to meet the multiplexing need for personalized therapy, it may also be useful to reduce the limits of detection (LOD) and generally increase the signal-to-noise ratio (SNR) in some clinical situations. Because specific lgE levels are patient specific and depend on age, total serum $\operatorname{lgE}$, and the time of year tested, some patients are incorrectly identified as 'negative' for allergic sensitization when the IgE level is below the detection threshold of existing technology (Hamilton and Williams 2010; Somville et al. 1989). Moreover, for point-of-care methods with capillary blood, the specimen may need to be diluted below 
the test instrument's lower limit of quantitation (Fan et al. 2008; Hamilton and Williams 2010). This requires a sensitive immunoassay. However, most commercial autoanalyzer systems used to measure specific IgE have detection limits in the 0.10 to $0.35 \mathrm{kU} / \mathrm{L}$ range $(1 \mathrm{U}=2.4 \mathrm{ng}$ ) (Hamilton ; Hamilton and Williams 2010; Wood et al.). Recently, fluorescence allergen microarrays have been utilized for allergy diagnosis. While mulitiplexing capability has been achieved, the detection sensitivity of fluorescence microarrays performed upon ordinary glass substrates can be improved upon by integrating a fluorescence enhancement mechanism via a photonic crystal surface. (Cretich et al. 2010; King et al. 2013; Skrindo et al. 2015). The ImmunoCAP ISAC assay (Phadia) is a commercially available microarray system for allergy diagnosis with a published detection limit of $0.35 \mathrm{kU} / \mathrm{L}$. To achieve the needed sensitivity, a platform with sufficient signal amplification, employing highly purified and/or recombinant allergens is needed (Linden et al.). Although other fluorescence enhancement methods have been reported (Fouqué et al. 2005; Volle et al. 2003), they either fail to provide stable and persistent enhancement due to quenching effects, or have low average enhancement due to sparse hot spot density. Meanwhile electrochemical assay methods have been demonstrated for detecting IgEs at concentration as low as $6 \mathrm{pM}$ (Salimi et al. 2014), the approach lacks a multiplexing capability, which limits its application in $\lg E$ detection. The PC substrate has demonstrated the ability to provide uniform, reproducible, and high average enhancement for high sensitivity detection of fluorescently tagged nucleic acid and protein molecules. 
Previously, we have demonstrated a photonic crystal enhanced fluorescence (PCEF) microarray system that can achieve high sensitivity for multiplexed cancer biomarker detection using low (10-20 $\mu$ l) sample volume (Ganesh et al. 2007; George et al. 2013; Huang et al. 2011). The protein microarray allows multiplexed detection and minimal reagent consumption, while the PC surface amplifies the fluorescence output and emission collection efficiency from the dye-tagged molecules through the use of narrow bandwidth optical resonances that are designed to occur at specific combinations of laser excitation wavelength and incident angle. The enhancement is provided by multiplying the independent effects of "PC enhanced excitation" and "PC enhanced extraction" as described in our previous publications (Ganesh et al. 2007; George et al. 2013; Huang et al. 2011). Briefly, PC enhanced excitation occurs by engineering a periodic nanostructured surface that functions as an optical resonator at the same wavelength as the laser that is used to excite a fluorescent dye. The amplified electric field associated with electromagnetic standing waves exists in an evanescent field region $\sim 100-200 \mathrm{~nm}$ above the PC surface (Ganesh et al. 2008), resulting in surface-bound fluorophores being exposed a greater degree of excitation power than they would experience on an unpatterned surface. PC enhanced extraction is achieved by designing the surface to simultaneously provide a second optical resonance at the wavelength of fluorophore emission, which is capable of efficiently extracting emitted photons preferentially in a direction normal to the surface, resulting in increased photon collection efficiency. The effects of the two phenomena are multiplicative and have been used to obtain up to 7500 -fold overall signal enhancement compared to an ordinary glass substrate (Pokhriyal et al. 2010). Although other fluorescence enhancement 
methods have been reported before (Fouqué et al. 2005; Volle et al. 2003), they either fail to provide stable and persistent enhancement due to quenching effect, or have low average enhancement because of the sparse 'hot' spots. The PC substrate, in contrast, can provide stable and high average enhancement and enable high sensitivity detection of proteins and DNAs. Our previous report showed that the immunoassay LOD for cancer biomarker detection was $0.3-10 \mathrm{pg} / \mathrm{mL}$ using the PC microarray system (Cunningham and Zangar 2012; George et al. 2013). The PCEF system is comprised of the PC nanostructured substrates, and a commercially available detection instrument. The PC surfaces are inexpensively manufactured from silicon wafers, using processes and equipment that are commonly applied to integrated circuit manufacturing (George et al. 2013). The detection instrument is aligned to efficiently couple illumination to the PC resonant mode, and to rapidly scan the laser across the PC surface to generate images of fluorescence emission intensity. The scanning instrument is capable of measuring large numbers of independent assays when capture molecules are printed on the PC to form an array, such as commonly used for DNA microarrays or protein microarrays (Mathias et al. 2008; Mathias et al. 2010).

In this work, we extend the application of the PCEF technology to a prototype allergy testing platform to achieve multiplexed, sensitive and specific $\lg E$ detection. This approach allows successful detection of allergen-specific $\lg E$ at low concentration, using small volumes of human serum. We found that our platform has higher sensitivity than a standard analyzer used for allergy immunoassays throughout the world (SILES and HSIEH 2011). In addition, the PCEF platform permits multiplexing of allergens, 
provides signal quantification, and detects several allergen-specific antibodies simultaneously on a single chip.

\section{Methods}

\subsection{PC Fabrication and Characterization}

The PC is comprised of a periodic surface structure fabricated in a low refractive index $(\mathrm{RI})$ silicon dioxide $\left(\mathrm{SiO}_{2}\right)$ layer on a silicon substrate (Figure 1(a)). The grating structure is coated with a high RI titanium dioxide $\left(\mathrm{TiO}_{2}\right)$ thin film. The PC has a period of $360 \mathrm{~nm}$, a duty cycle of $36 \%$, a grating depth of $40 \mathrm{~nm}$, and a $\mathrm{TiO}_{2}$ thickness of $130 \mathrm{~nm}$. A commercial vendor (Novati Technologies Inc., Austin TX) was contracted for performing photolithography and reactive ion etching (RIE) of the $\mathrm{SiO}_{2}$ grating structure over 8-inch diameter wafers, while $\mathrm{TiO}_{2}$ thin films were deposited upon whole wafers at a second vendor (Intlvac Inc., Niagara Falls NY). Following lithography, etching, and $\mathrm{TiO}_{2}$ deposition, the wafers were diced into $1.0 \times 0.5$ inch $^{2}$ pieces. An SEM image showing the surface structure of the PC is presented in the inset of Figure 1 (b).

The PC is designed to enhance the fluorescent intensity of Cy5 dye though the enhanced excitation and enhanced extraction mechanisms. The enhanced excitation can be achieved by illuminating the PC at a specific incident angle for a given laser illumination wavelength (Ganesh et al. 2007). At the resonance coupling condition of the $\mathrm{PC}$, the enhanced electric fields are confined to the surface of the device and extend into the adjacent media with exponentially decaying intensity, and thus only surfacebound fluorophores will be exposed to enhanced energy from the laser. The PC resonant coupling can be observed by measuring the reflected intensity of laser illumination as a function of incident angle, as shown in Figure 1(c), where the peak 
reflected intensity corresponds to the angle at which optimal coupling occurs. Here, an incident angle of $4.12^{\circ}$ achieves the resonance condition. Our previous work demonstrated that the PC also provides a second resonance at $\lambda \sim 685 \mathrm{~nm}$ that provides an enhanced extraction effect (Wu et al. 2010).

\subsection{Source of materials.}

We evaluated the feasibility of PCEF for selective characterization of the presence of allergen-specific lgE using three different allergen materials (Timothy grass extract and cat hair \& epithelium extract (Hycor Biomedical, Garden Grove, CA) and Fel d1, a highly purified protein from cat extract (Indoor Biotechnologies, Charlottesville, VA). The human sera used in these studies were provided by ViracorIBT Laboratories (Lee's Summit, MO )and Hycor Biomedical, Inc. Our collaborators at Hycor provide us with the negative control which is clinically tested true negative sample. These discard sera had previously been tested for various specific IgEs with a standard clinical analyzer used for allergy testing (ImmunoCAP, ThermoFisher, Fremont CA).

\subsection{Preparation and allergen printing on the PC surface.}

A single $0.5 \times 1.0 \mathrm{in}^{2} \mathrm{PC}$ die holds 10 subarrays, and each subarray contains 4 sets of 4 replicate spots per protein for a total of 16 spots. Before allergen printing, the PC surface was cleaned and activated with a vapor-phase epoxysilane process. The epoxysilane chemistry was chosen for its low background fluorescence (Dorvel et al. 2009) and high binding capacity to capture antibodies (Zhu et al. 2000). The devices were first cleaned by sonication in 2" petri dishes of acetone, isopropanol, and deionozied (DI) water for 2 minutes each. The devices were then dried in a stream of 
$\mathrm{N}_{2}$ and then treated in an oxygen plasma system (Diener, Pico) for 10 minutes (power of $100 \mathrm{~W}$, pressure of $0.75 \mathrm{mTorr})$. The backside of each device was then adhered to the inside of a screw top lid of a 2" glass container. At the base of the container, 100 $\mu \mathrm{L}$ of (3-Glycidoxypropyl) trimethoxysilane (GPTS, Sigma Aldrich, Saint Louis, MO) was placed and the screw top lid was securely placed over the dish. After securely tightening the lids, each dish with a device adhered to its lid was placed in a vacuum oven for an overnight incubation at a temperature of $80{ }^{\circ} \mathrm{C}$ and a pressure of 30 Torr. The devices were then detached from the lids and sonicated in 2" petri dishes of toluene, methanol, and DI water for 2 minutes each and dried under a stream of $\mathrm{N}_{2}$.

The allergen microarrays were printed by a commercially available spot printing service (Arraylt) using their instrument (Arrayit NanoPrint LM60 Microarrayer) which provides a controlled environment (ambient temperature and $50 \%$ relative humidity). Four pins (946MP3 Microarray Printing Pins) were used to print the sixteen spots. Printing pins were cleaned between sample pickups with 15 s sonication, 4 cycles of washing $(2.5 \mathrm{~s})$ in DI water and drying (1 s). Measured spot diameters were $79.00 \pm$ $2.22 \mu \mathrm{m}$. Row spacing was $149.25 \pm 3.26 \mu \mathrm{m}$ and column spacing was $200.75 \pm 0.82$ $\mu \mathrm{m}$. To assure the protein stability, the arrays were stored in a cool, low humidity environment in which prior experience has demonstrated that printed proteins remain active for more than one month.

Proteins deposited onto solid surfaces display distinct characteristics due to differences in charge, molecular structure, acidity, specificity, affinity, hydrophobicity and stability. The diversity of protein structures in allergen extracts poses an additional challenge for identifying a universal assay surface and the ideal solution conditions 
( e.g. probe concentration, buffer composition, $\mathrm{pH}$, incubation times, etc.) that best maintain capture protein functionality equally for all the probe molecules in a microarray (Angenendt et al. 2003; Seurynck-Servoss et al. 2007). PBS spotting buffer was specified by the array printing commercial service (Arraylt) through their prior experience printing similar reagents. In this work, we evaluated the optimal spotting concentration by spotting with different concentrations of allergen solutions. Two allergen extracts (cat and Timothy grass) and a highly purified cat allergen (Fel d1) were used to produce the probe spots. Fel $\mathrm{d} 1$ is the major allergen in cat hair and epithelium extracts, and is expected to have better sensitivity and specificity than the crude cat extracts, which are complex mixtures of proteins. We occasionally observed spots in the array that were either missing or extremely weak, which is attributed to errors that occurred during the printing process.

\subsection{Testing Procedures}

The slides were placed in a 10-well custom-made slide module assembly (Figure 3(b)) where each well can hold a $10 \mu \mathrm{L}$ test sample. The assay procedure is illustrated in Figure 2. The arrays were blocked with a blocking buffer that contains $10 \mathrm{mM}$ sodium phosphate, $0.15 \mathrm{M} \mathrm{NaCl}, 0.05 \%(\mathrm{v} / \mathrm{v})$ Tween-20, $1 \%(\mathrm{w} / \mathrm{v})$ Human Serum Albumin, 1\%(v/v) ProClin 950 (Hycor Biomedical, Inc.) for 1h. The wash buffer was supplied by co-authors at Hycor. Proclin 950 is the preservative used in their commercial assay, as an alternative to sodium azide, which may interfere with HRP conjugates typically used for ELISA assays. All incubations were performed at room temperature. The arrays were then washed five times with a wash buffer that contains 4.5\% (w/v) NaCl, 0.25\% (v/v) Tween-20, 15\% (v/v) Propylene glycol and 0.05\% (v/v) 
ProClin 950 (Hycor Biomedical, Inc.). Next, each well was incubated with $10 \mu \mathrm{L}$ human serum or serum dilution in blocking buffer overnight. This was followed by five rinses after which the PC surface was incubated with only $10 \mu \mathrm{L}$ of the biotinylated detection antibody mixture (mouse monoclonal B3102E8 anti-human IgE and mouse monoclonal HP8029 anti-human IgE diluted in PBS, at the mixing ratio of 1:1, Abcam Inc.) in each well of the slide module assembly for 4 hours. The PC substrates were then washed five times followed by incubation with a $1 \mu \mathrm{g} / \mathrm{mL}$ solution of Cy5conjugated streptavidin (diluted in PBS, Invitrogen) for 30 minutes. Finally, the devices were washed 5 times and dried in an ambient environment.

\subsection{Image and Data Acquisition}

The substrates were scanned with a commercially available confocal laser microarray scanner (Tecan LS Reloaded). This scanner was fitted with a $632.8 \mathrm{~nm} 5$ $\mathrm{mW}$ laser for Cy5 excitation and a Cy5 emission filter (bandpass, 670-715nm). The incident light was TM polarized and made incident on the substrates at an angle of $4.12^{\circ}$ so that maximum laser coupling efficiency could be achieved. Scans were obtained at a resolution of $10 \mu \mathrm{m}$ and the photomultiplier tube (PMT) gain was adjusted to 80 so that the largest fluorescence intensities did not saturate the PMT. Fluorescent images were analyzed using ImageJ to compute spot and local background intensities as well as standard deviations for each spot. In order to mitigate small differences in spot diameter between printed assays, caused by variability in viscosity and hydrophilicity from one material to another, a $50 \mu \mathrm{m}$ diameter circular region from the center of each spot was selected as the region of interest, where the medium fluorescence intensity value was used to represent the spot intensity. In some arrays 
reported in this work, a row "location spots" of Alexa-fluor dye were printed to help visually identify and orient the array after scanning. The location spots consistently did not spread laterally as far as the printed antigen materials, although a $50 \mu \mathrm{m}$ diameter circle is still used to mark their location.

\section{Results}

\subsection{Selectivity of the Assay}

We constructed a testing well on the PC chip by creating 4-plexed allergen arrays for simultaneous allergen-specific lgE antibody detection with low sample volumes (10 $\mu \mathrm{L})$. As shown in Figure 3(a), the first row is cat hair extract, the second row is Fel d1, the third row is Timothy grass extract and the last row is Alexa-Fluor-555 fluorescencent streptavidin conjugates to identify location of protein spots (Life Technologies). AlexaFluor-555 fluorescencent streptavidin (SA-Alexa) conjugates were used to print the location spots, which has weaker binding to the aminosilane surface compared to allergen molecules. Therefore, we observed much smaller spot size for the location spots.

To evaluate the ability of our platform to detect specific $\lg E$ in a variety of different sera, we obtained samples that had been tested with a standard clinical immunoassay analyzer. Approximately $10 \mu \mathrm{L}$ of each serum or dilution was added to the PC array surface. After processing, we analyzed the chips in a microarray scanner to detect and quantify the signals. Some of the rows of spots had high variances across spots after quantifying the fluorescence images. This issue is possibly related 
to problems with sample application and washing the prototype platform or with allergen extract variability.

The assays exhibited selectivity with these sera. If the serum contained specific IgE antibodies against one or more allergens, we detected positive signals specifically on the respective allergen spots. With this technique, we were able to distinguish the serum samples with different amount of grass-pollen and Fel d1 specific antibodies (Figure 3(c)). For example, as shown in Figure 3(d), we detected strong fluorescence on grass pollen spots for a 4-fold dilution of a serum which had high level grass-pollen specific IgE antibodies, while the fluorescence was not detected for a 4-fold dilution of a serum that had a specific $\lg E$ concentration less than the $0.1 \mathrm{kU} / \mathrm{L}$ test threshold. In addition, we observed fluorescence from Fel d1 spots for both sera that were previously shown by the reference method to contain $\lg E$ antibodies against Fel $\mathrm{d} 1$. No fluorescence was detected for either grass pollen, cat or Fel d1 spots in the cases of negative serum incubation (or buffer only).

\subsection{Sensitivity of the PCEF Array System}

Two human sera that contain cat hair-specific $\lg E$ were tested over a range of 8 concentrations in a 2-fold dilution series. To assess the sensitivity of IgE testing by PC microarray, the original sera were also tested by ImmunoCAP, the reference method widely used for allergy testing (Hamilton et al. 2011; Jacquenet et al. 2009).

The fluorescent images of microspots at different antibody concentrations are presented in Figure 4. Note that a small number of replicate spots (indicated with a red " $x$ " through the spot) were excluded from analysis. We found that printed spot density

occasionally, and randomly, produced capture spots of anomalously low density, 
which in turn resulted in fluorescent spot intensities substantially lower than neighboring replicate spots. We implemented an algorithm in which a spot with less than $1 / 10$ the intensity of the mean intensity of all four replicates would be designated as a "bad spot" and excluded from calculation of average intensity or standard deviation. Even after excluding bad spots by this method, all assays are represented by at least three replicate spots. Note that Fel d1 spots have higher fluorescence intensities than cat extract spots for the same serum dilution, which confirms that purified allergen has stronger binding capacity (i.e. affinity, antigen density on solid phase, etc.) and thus higher apparent sensitivity than the crude extracts. To quantitatively characterize how easily a spot can be distinguished from the noise, we define signal to noise ratio (SNR) as the net signal intensity divided by the standard deviation of the background intensity. A spot with SNR larger than 3 is regarded as detectable over the background noise. Figure 4 shows that all of the fel d1 spots were detectable over the $0.005-4.000 \mathrm{kU} / \mathrm{L}$ range. The average SNR over four fel $\mathrm{d} 1$ replicates at the lowest concentration $(0.005 \mathrm{kU} / \mathrm{L})$ is 5.16 . However, in order to estimate the lowest detectable concentration, we performed a dose response study and compared fluorescence intensities at different concentrations with the background intensity.

The signal intensities from each dilution in the concentration series were used to generate a standard curve (Figure 5) for the Fel d1 allergen using Origin (Northampton, MA). There are four replicate spots for each assay. Error bars representing one standard deviation for each assay are plotted on the standard curve (Figure 5) to show the intra assay reproducibility. The limit of detection (LOD) is defined as the 
concentration corresponding to the blank intensity (i.e., the intensity of the negative control spot of diluent) plus 3 standard deviations from all assay spots. Negative controls performed by exposing the capture allergens to a negative serum sample resulted in no observable fluorescence signal above the surrounding area. The black solid line in the inset represents the background intensity (blank intensity plus 3 standard deviations), while the dashed line indicates the intensity from the negative control spots. From the inset of Figure 5, we can tell that fluorescence intensities from Fel d1 spots assayed with diluted sera with calculated antibody concentrations of 0.005 $\mathrm{kU} / \mathrm{L}$ and $0.011 \mathrm{kU} / \mathrm{L}$ were below the background intensity although they were observable in the image. Therefore, we estimated the LOD value for antibody specific to Fel $\mathrm{d} 1$ is $\sim 0.02 \mathrm{kU} / \mathrm{L}$. This is lower than the lowest detectable concentration of $0.1 \mathrm{kU} / \mathrm{L}$ measured by the ImmunoCAP system.

We can also use this data to estimate the Lower Limit of Quantitation (LLOQ). According to FDA guidelines for industry bioanalytical method validation, LLOQ must meet the following two conditions: (1) The analyte response at LLOQ must be at least 5 times the response noise above the blank response; (2) CV must be lower than $20 \%$. In our case, we measure a mean blank intensity of 38 counts and standard deviation of the blank intensity of 60 counts, therefore response at LLOQ must be larger than $\left(\right.$ mean $\left.+5^{*} \mathrm{std}=\right) 338$. The lowest concentration that gives intensity larger than 338 and also has a CV of $(69 / 852=) 8 \%$ is $0.1 \mathrm{kU} / \mathrm{L}$, which is determined as the LLOQ for our approach. With regard to the model allergen used, PCEF technology appears to be more sensitive than the ImmunoCAP system. 


\section{Conclusion}

Allergy blood tests measure levels of lgE against specific allergens such as foods, inhalants, medications, latex and venoms. These tests can confirm the diagnosis of an allergy disorder, supplementing a clinical history that is consistent with an immediate allergic reaction. The detection of specific IgE to some food or inhalant allergens by $\lg E$ requires a senstitive detection platform since $\lg E$ constitutes the least abundant immunoglobulin. In addition, multiplexing capability is needed to fulfill the requirements for component-resolved diagnostics and personalized therapies.

In this work, a PCEF microarray platform successfully detected lowconcentration IgE in human sera. The PC surface, designed to provide optical resonances for the excitation wavelength and emission wavelength of Cy5, was used to amplify the fluorescence signal intensity measured from a multiplexed protein microarray. Comparison of the LOD measured by a commercially available antibody analyzer to the PC microarray system demonstrates that the PCEF microarray system provides lower limits of detection. The dose-response data shown for this prototype assay had an LOD $\sim 0.02 \mathrm{kU} / \mathrm{L}$ for Fel d1 specific lgE. The clinical cutoff for this assay is at $0.35 \mathrm{kU} / \mathrm{L}$ for a specific allergen in a non-allergic person. Thus, an LoD below 0.1 $\mathrm{kU} / \mathrm{L}$ is considered to be an excellent value for clinical diagnostics. In addition to the high sensitivity, the PCEF microarray platform allows simultaneous detection and quantification of antibodies to various allergens. While most ImmunoCAP testing instruments require milliliters of blood, the microarray platform requires only $10 \mu \mathrm{L}$ of serum, which offers compatibility with less invasive sample collection via finger-prick. Finally, our results demonstrate the efficacy of using purified allergen components as 
the selective capture agent, which delivered greater sensitivity and reproducibility than raw allergen extracts.

In addition to addressing a current clinical need for improved IgE testing options, we believe that this technology could enable a range of advances in clinical allergy testing. For example, serial monitoring of $\lg E$ and $\lg G$ antibodies in patients undergoing current allergen immunotherapy treatment protocols might be used to assess the effectiveness of the therapy. The measurement of cytokines and other mediators of allergic inflammation (e.g. eosinophil mediators) can also be done with this technology. Ultimately, we believe that this technology could be deployed to facilitate diagnostic screening, immunologic characterization and monitoring of many autoimmune diseases including celiac disease, systemic lupus erythematosis, myasthenia gravis, thyroid disease, etc.

\section{Acknowledgement}

The authors gratefully acknowledge Scott McDonald and David Switzer at machine shop of ECE department for their help making the 10-well assembly.

This work was supported by grants from the National Institutes of Health (GM086382A) and the National Science Foundation (CBET 07-54122). Any opinions, findings, conclusions, or recommendations expressed in this material are those of the authors and do not necessarily reflect the views of the National Institutes of Health or the National Science Foundation.

\section{References}


Angenendt, P., Glökler, J., Sobek, J., Lehrach, H., Cahill, D.J., 2003. Next generation of protein microarray support materials:: Evaluation for protein and antibody microarray applications. Journal of Chromatography A 1009(1-2), 97-104.

Burks, A.W., Jones, S.M., Boyce, J.A., Sicherer, S.H., Wood, R.A., Assa'ad, A., Sampson, H.A., 2011. NIAIDSponsored 2010 Guidelines for Managing Food Allergy: Applications in the Pediatric Population. Pediatrics 128(5), 955-965.

Chapman, M.D., Rowntree, S., Mitchell, E.B., Di Prisco de Fuenmajor, M.C., Platts-Mills, T.A.E., 1983. Quantitative assessments of $\operatorname{IgG}$ and IgE antibodies to inhalant allergens in patients with atopic dermatitis. Journal of Allergy and Clinical Immunology 72(1), 27-33.

Conroy, M.E., 2013. Food Allergy: Review of Epidemiology, Risk Factors, and Future Treatments. American Journal of Lifestyle Medicine.

Cunningham, B.T., Zangar, R.C., 2012. Photonic crystal enhanced fluorescence for early breast cancer biomarker detection. Journal of Biophotonics 5(8-9), 617-628.

Dorvel, B., Reddy, B., Block, I., Mathias, P., Clare, S.E., Cunningham, B., Bergstrom, D.E., Bashir, R., 2009. Vapor-Phase Deposition of Monofunctional Alkoxysilanes for Sub-Nanometer-Level Biointerfacing on Silicon Oxide Surfaces. Advanced Functional Materials 20(1), 87-95.

Fan, R., Vermesh, O., Srivastava, A., Yen, B.K.H., Qin, L., Ahmad, H., Kwong, G.A., Liu, C.-C., Gould, J., Hood, L., Heath, J.R., 2008. Integrated barcode chips for rapid, multiplexed analysis of proteins in microliter quantities of blood. Nat Biotech 26(12), 1373-1378.

Feeney, N., Barrett, N., Philip, R., 2012. 483 Regional Review of Paediatric Radioallergosorbent Tests ${ }^{\circledR}$ (Rast) over 5 Years in Ireland: an Epidemiological Study in a Defined Birth Cohort. Archives of Disease in Childhood 97(Suppl 2), A141.

Fouqué, B., Schaack, B., Obeïd, P., Combe, S., Gétin, S., Barritault, P., Chaton, P., Chatelain, F., 2005. Multiple wavelength fluorescence enhancement on glass substrates for biochip and cell analyses. Biosensors and Bioelectronics 20(11), 2335-2340.

Ganesh, N., Mathias, P.C., Zhang, W., Cunningham, B.T., 2008. Distance dependence of fluorescence enhancement from photonic crystal surfaces. Journal of Applied Physics 103(8), -.

Ganesh, N., Zhang, W., Mathias, P.C., Chow, E., Soares, J.A.N.T., Malyarchuk, V., Smith, A.D., Cunningham, B.T., 2007. Enhanced fluorescence emission from quantum dots on a photonic crystal surface. Nat Nano 2(8), 515-520.

George, S., Chaudhery, V., Lu, M., Takagi, M., Amro, N., Pokhriyal, A., Tan, Y., Ferreira, P., Cunningham, B.T., 2013. Sensitive detection of protein and miRNA cancer biomarkers using silicon-based photonic crystals and a resonance coupling laser scanning platform. Lab on a Chip 13(20), 4053-4064.

Gergen, P.J., Turkeltaub, P.C., Kovar, M.G., 1987. The prevalence of allergic skin test reactivity to eight common aeroallergens in the U.S. population: Results from the second National Health and Nutrition Examination Survey. Journal of Allergy and Clinical Immunology 80(5), 669-679.

Hagy, G.W., Settipane, G.A., 1971. Prognosis of positive allergy skin tests in an asymptomatic population: A three year follow-up of college students. Journal of Allergy and Clinical Immunology 48(4), 200-211.

Hamilton, R.G., Allergic sensitization is a key risk factor for but not synonymous with allergic disease. Journal of Allergy and Clinical Immunology 134(2), 360-361.

Hamilton, R.G., Mudd, K., White, M.A., Wood, R.A., 2011. Extension of food allergen specific IgE ranges from the ImmunoCAP to the IMMULITE systems. Annals of Allergy, Asthma \& Immunology 107(2), 139144.

Hamilton, R.G., Williams, P.B., 2010. Human IgE antibody serology: A primer for the practicing North American allergist/immunologist. Journal of Allergy and Clinical Immunology 126(1), 33-38.

Harwanegg, C., Laffer, S., Hiller, R., Mueller, M.W., Kraft, D., Spitzauer, S., Valenta, R., 2003. Microarrayed recombinant allergens for diagnosis of allergy. Clinical \& Experimental Allergy 33(1), 7-13. 
Howanitz, P.J., 2005. Errors in Laboratory Medicine: Practical Lessons to Improve Patient Safety. Archives of Pathology \& Laboratory Medicine 129(10), 1252-1261.

Huang, C.-S., George, S., Lu, M., Chaudhery, V., Tan, R., Zangar, R.C., Cunningham, B.T., 2011. Application of Photonic Crystal Enhanced Fluorescence to Cancer Biomarker Microarrays. Analytical Chemistry 83(4), 1425-1430.

Jacquenet, S., Morisset, M., Battais, F., Denery-Papini, S., Croizier, A., Baudouin, E., Bihain, B., MoneretVautrin, D.A., 2009. Interest of ImmunoCAP System to Recombinant $\omega-5$ Gliadin for the Diagnosis of Exercise-Induced Wheat Allergy. International Archives of Allergy and Immunology 149(1), 74-80.

Jahn-Schmid, B., Harwanegg, C., Hiller, R., Bohle, B., Ebner, C., Scheiner, O., Mueller, M.W., 2003. Allergen microarray: comparison of microarray using recombinant allergens with conventional diagnostic methods to detect allergen-specific serum immunoglobulin E. Clinical \& Experimental Allergy 33(10), 1443-1449.

Jutel, M., Jaeger, L., Suck, R., Meyer, H., Fiebig, H., Cromwell, O., 2005. Allergen-specific immunotherapy with recombinant grass pollen allergens. Journal of Allergy and Clinical Immunology 116(3), 608-613.

Kazemi-Shirazi, L., Pauli, G., Purohit, A., Spitzauer, S., Fröschlc, R., Hoffmann-Sommergruber, K., Breiteneder, H., Scheiner, O., Kraft, D., Valenta, R., 2000. Quantitative IgE inhibition experiments with purified recombinant allergens indicate pollen-derived allergens as the sensitizing agents responsible for many forms of plant food allergy. Journal of Allergy and Clinical Immunology 105(1, Part 1), 116-125.

Lieberman, J., Sicherer, S., 2011. Diagnosis of Food Allergy: Epicutaneous Skin Tests, In Vitro Tests, and Oral Food Challenge. Curr Allergy Asthma Rep 11(1), 58-64.

Linden, C.C., Misiak, R.T., Wegienka, G., Havstad, S., Ownby, D.R., Johnson, C.C., Zoratti, E.M., Analysis of allergen specific IgE cut points to cat and dog in the Childhood Allergy Study. Annals of Allergy, Asthma \& Immunology 106(2), 153-158.e152.

Mathias, P.C., Ganesh, N., Cunningham, B.T., 2008. Application of Photonic Crystal Enhanced Fluorescence to a Cytokine Immunoassay. Analytical Chemistry 80(23), 9013-9020.

Mathias, P.C., Jones, S.I., Wu, H.-Y., Yang, F., Ganesh, N., Gonzalez, D.O., Bollero, G., Vodkin, L.O., Cunningham, B.T., 2010. Improved Sensitivity of DNA Microarrays Using Photonic Crystal Enhanced Fluorescence. Analytical Chemistry 82(16), 6854-6861.

Östblom, E., Lilja, G., Ahlstedt, S., Van Hage, M., Wickman, M., 2008. Patterns of quantitative foodspecific IgE-antibodies and reported food hypersensitivity in 4-year-old children. Allergy 63(4), 418-424.

Pipkorn, U., Hammarlund, A., EnerbÄCk, L., 1989. Prolonged treatment with topical glucocorticoids results in an inhibition of the allergen-induced weal-and-flare response and a reduction in skin mast cell numbers and histamine content. Clinical \& Experimental Allergy 19(1), 19-25.

Pokhriyal, A., Lu, M., Chaudhery, V., Huang, C.-S., Schulz, S., Cunningham, B.T., 2010. Photonic crystal enhanced fluorescence using a quartz substrate to reduce limits of detection. Opt. Express 18(24), 24793-24808.

Rudenko, M., Seneviratne, S., Boyle, R., Burova, K., Egner, W., Bhayat-Cammak, A., Bradshaw, N., 2013. Immunocap ISAC as an important diagnostic tool in rhino-sinusitis. Clinical and Translational Allergy 3(Suppl 2), P32.

Sampson, H.A., Ho, D.G., 1997. Relationship between food-specific IgE concentrations and the risk of positive food challenges in children and adolescents. Journal of Allergy and Clinical Immunology 100(4), 444-451.

Schellenberg, R.R., Adkinson, N.F., 1975. Measurement of Absolute Amounts of Antigen-Specific Human IgE by a Radioallergosorbent Test (RAST) Elution Technique. The Journal of Immunology 115(6), 15771583.

Seurynck-Servoss, S.L., White, A.M., Baird, C.L., Rodland, K.D., Zangar, R.C., 2007. Evaluation of surface chemistries for antibody microarrays. Analytical Biochemistry 371(1), 105-115. 
Sicherer, S.H., Wood, R.A., ALLERGY, t.S.O., IMMUNOLOGY, 2012. Allergy Testing in Childhood: Using Allergen-Specific IgE Tests. Pediatrics 129(1), 193-197.

SILES, R.I., HSIEH, F.H., 2011. Allergy blood testing: A practical guide for clinicians. Cleveland Clinic Journal of Medicine 78(9), 585-592.

Simpson, A., Soderstrom, L., Ahlstedt, S., Murray, C.S., Woodcock, A., Custovic, A., IgE antibody quantification and the probability of wheeze in preschool children. Journal of Allergy and Clinical Immunology 116(4), 744-749.

Somville, M.A., Machiels, J., Gilles, J.G.G., Saint-Remy, J.-M.R., 1989. Seasonal variation in specific IgE antibodies of grass-pollen hypersensitive patients depends on the steady state IgE concentration and is not related to clinical symptoms. Journal of Allergy and Clinical Immunology 83(2, Part 1), 486-494.

Turkeltaub, P.C., Gergen, P.J., 1989. The risk of adverse reactions from percutaneous prick-puncture allergen skin testing, venipuncture, and body measurements: Data from the second National Health and Nutrition Examination Survey 1976-1980 (NHANES II). Journal of Allergy and Clinical Immunology 84(6, Part 1), 886-890.

Valenta, Lidholm, Niederberger, Hayek, Kraft, GrÖNlund, 1999. The recombinant allergen-based concept of component-resolved diagnostics and immunotherapy (CRD and CRIT). Clinical \& Experimental Allergy 29(7), 896-904.

Volle, J.N., Chambon, G., Sayah, A., Reymond, C., Fasel, N., Gijs, M.A.M., 2003. Enhanced sensitivity detection of protein immobilization by fluorescent interference on oxidized silicon. Biosensors and Bioelectronics 19(5), 457-464.

Wahyuni, S., Van Ree, R., Mangali, A., Supali, T., Yazdanbakhsh, M., Sartono, E., 2003. Comparison of an enzyme linked immunosorbent assay (ELISA) and a radioallergosorbent test (RAST) for detection of IgE antibodies to Brugia malayi. Parasite Immunology 25(11-12), 609-614.

Wills-Karp, M., Santeliz, J., Karp, C.L., 2001. The germless theory of allergic disease: revisiting the hygiene hypothesis. Nat Rev Immunol 1(1), 69-75.

Wood, R.A., Segall, N., Ahlstedt, S., Williams, P.B., Accuracy of IgE antibody laboratory results. Annals of Allergy, Asthma \& Immunology 99(1), 34-41.

Wu, H.-Y., Zhang, W., Mathias, P.C., Cunningham, B.T., 2010. Magnification of photonic crystal fluorescence enhancement via TM resonance excitation and TE resonance extraction on a dielectric nanorod surface. Nanotechnology 21(12), 125203.

Yazdanbakhsh, M., Kremsner, P.G., van Ree, R., 2002. Allergy, Parasites, and the Hygiene Hypothesis. Science 296(5567), 490-494.

Zhu, H., Klemic, J.F., Chang, S., Bertone, P., Casamayor, A., Klemic, K.G., Smith, D., Gerstein, M., Reed, M.A., Snyder, M., 2000. Analysis of yeast protein kinases using protein chips. Nature genetics $26(3), 283-$ 290.

Zuberbier, T., Lötvall, J., Simoens, S., Subramanian, S.V., Church, M.K., 2014. Economic burden of inadequate management of allergic diseases in the European Union: a GA2LEN review. Allergy, n/a-n/a. 
(a)

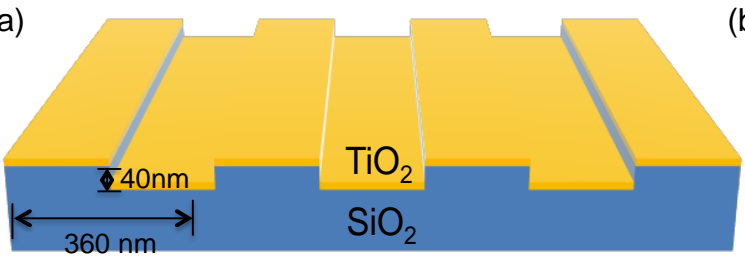

(c)

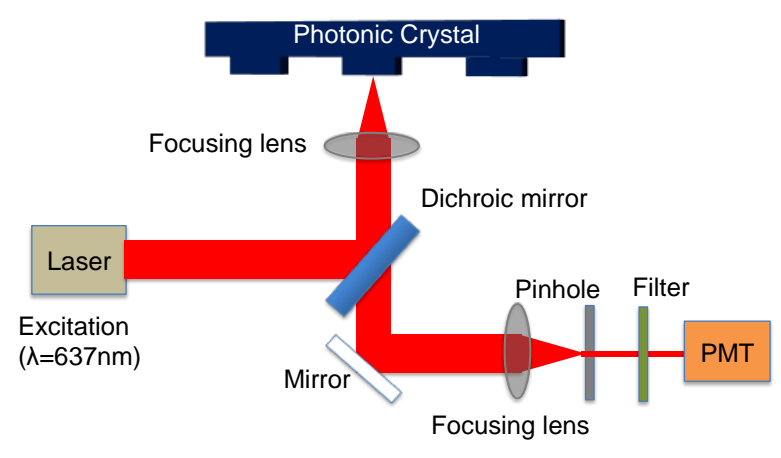

(b)

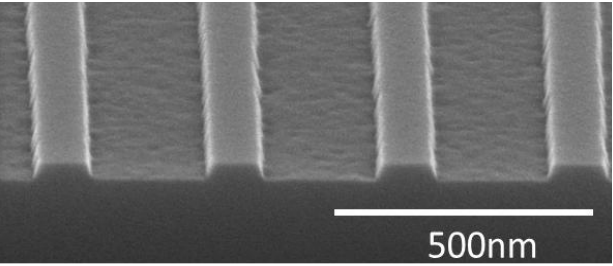

(d)

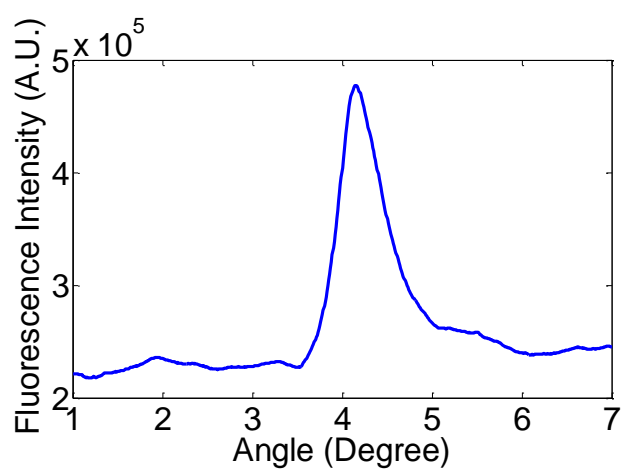

Figure 1. (a) Schematic of the Photonic Crystal (PC) structure and the laser scanning detection instrument. The PC is comprised of a periodic surface structure fabricated in a low refractive index (RI) silicon dioxide $\left(\mathrm{SiO}_{2}\right)$ layer on a silicon substrate that is overcoated with a thin film of high refractive index $\mathrm{TiO}_{2}$. (b) An SEM image showing the surface structure of the PC. (c) Schematic diagram of the detection instrument. (d) Reflected intensity as a function of incident angle of the PC when it is illuminated by a Transverse Magnetic (TM) polarized laser at a wavelength of $\lambda=637 \mathrm{~nm}$. The peak location of the spectrum indicates the resonance condition is achieved at the incident angle of $4.12^{\circ}$. 


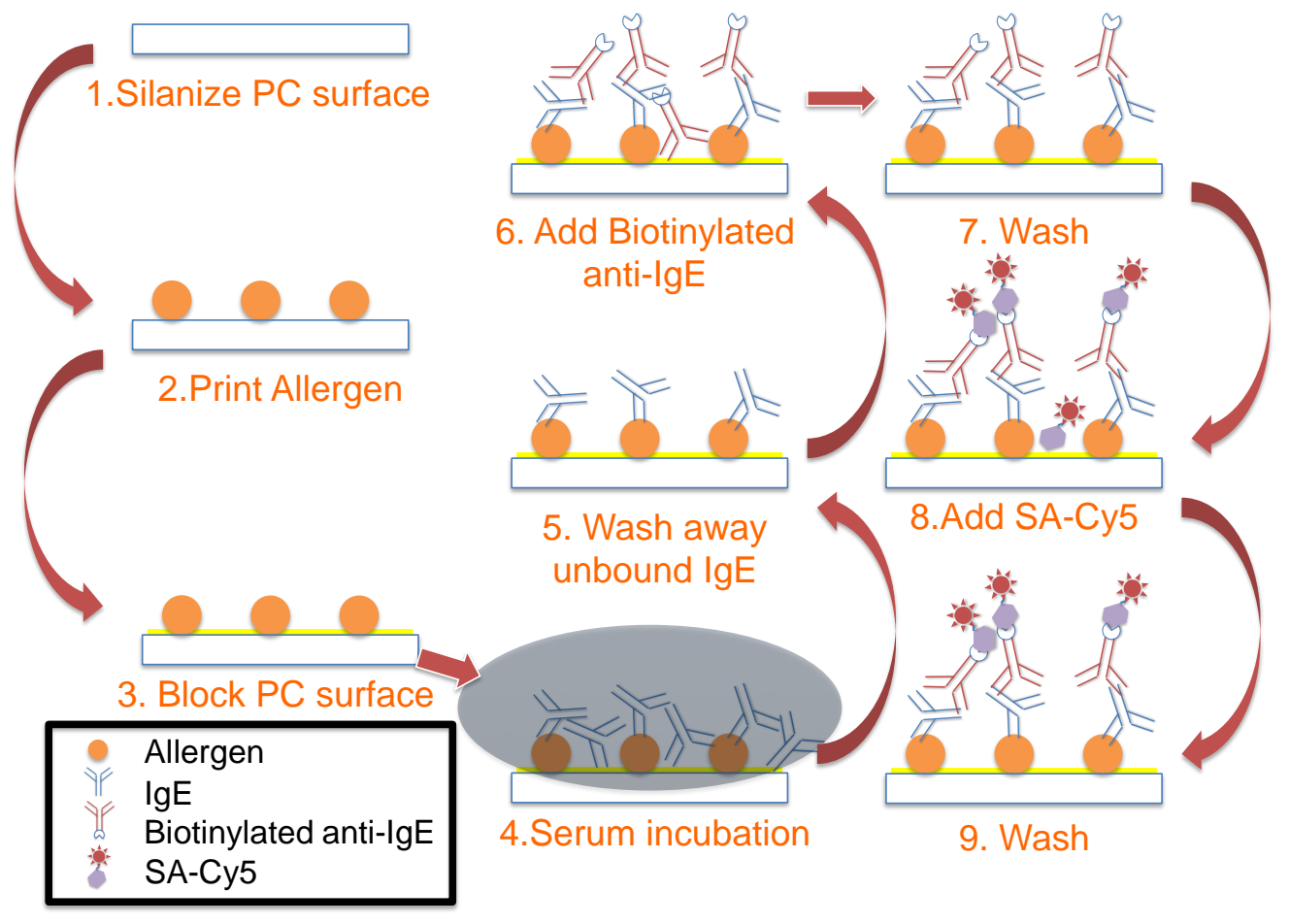

Figure 2. Schematic diagram illustrating the major assay steps. A PC surface was first silanized, after which allergens were printed in the form of micro-spots. The PC surface was subsequently blocked to prevent any further protein binding to the surface. Human serum containing IgE was incubated and unbound IgE was removed. Next, biotinylated anti-IgE was added as the detection antibody. Following the wash step, SA-Cy5 was added as the fluorescent tag. 
(a)

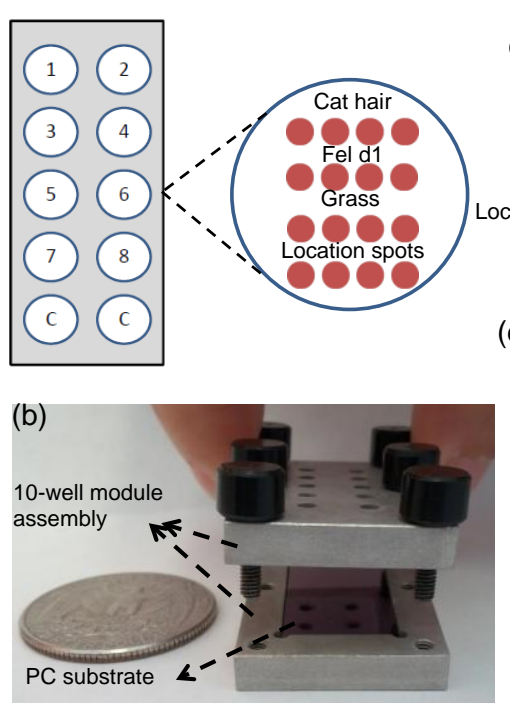

(c)

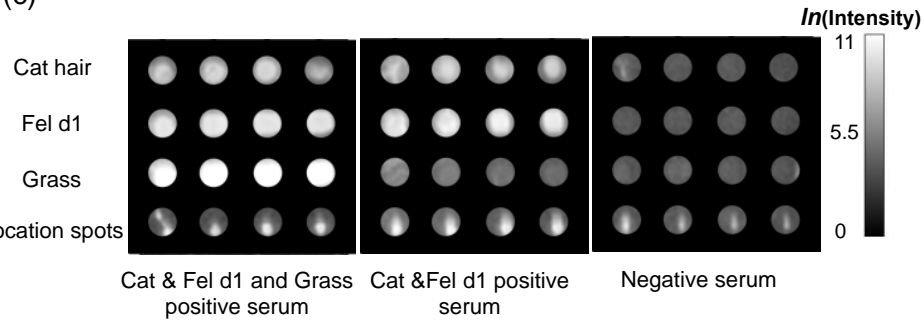

(d)

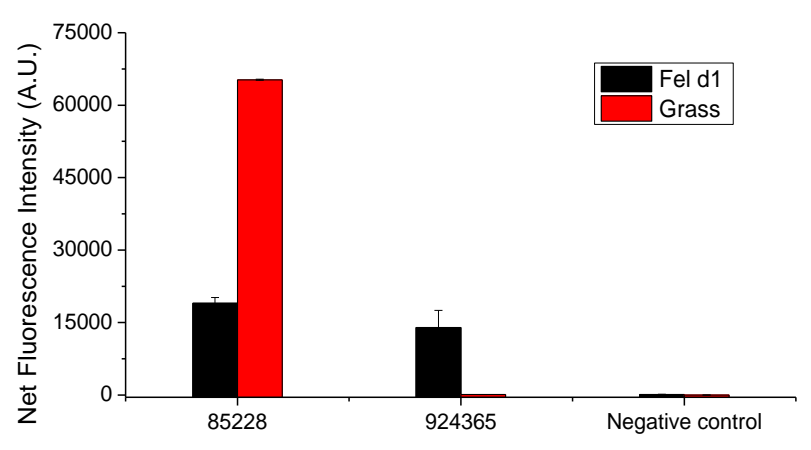

Figure 3. (a) A PC holds 10 subarrays, and each subarray contains 4 sets of 4 replicate spots per protein for a total of 16 spots. Inset: schematic of the microarray layout. The first row is the cat hair extract, the second row is the Fel d1, the third row is the Timothy grass extract and the last row is a set of array location fluorescent spots comprised of AlexaFluor-555 fluorescencent streptavidin conjugates. (b) A 10 well format custom-made slide module assembly in which the PC is inserted during the assay steps. (c) Fluorescence images of the arrays tested with different sera. (d) Average fluorescence intensities from Fel $\mathrm{d} 1$ and Timothy grass spots. The result indicates that the fel $\mathrm{d} 1$ and grass pollen assays exhibited excellent selectivity. Strong fluorescence was observed on grass pollen spots for 4 -fold dilution of serum \#85228 which had a high concentration of grass-pollen specific antibodies, while no fluorescent signal was observed for 4 -fold dilution of serum \#924365 that had low concentration. No fluorescence was detected on either grass extract or Fel d1 spots for the negative serum. 

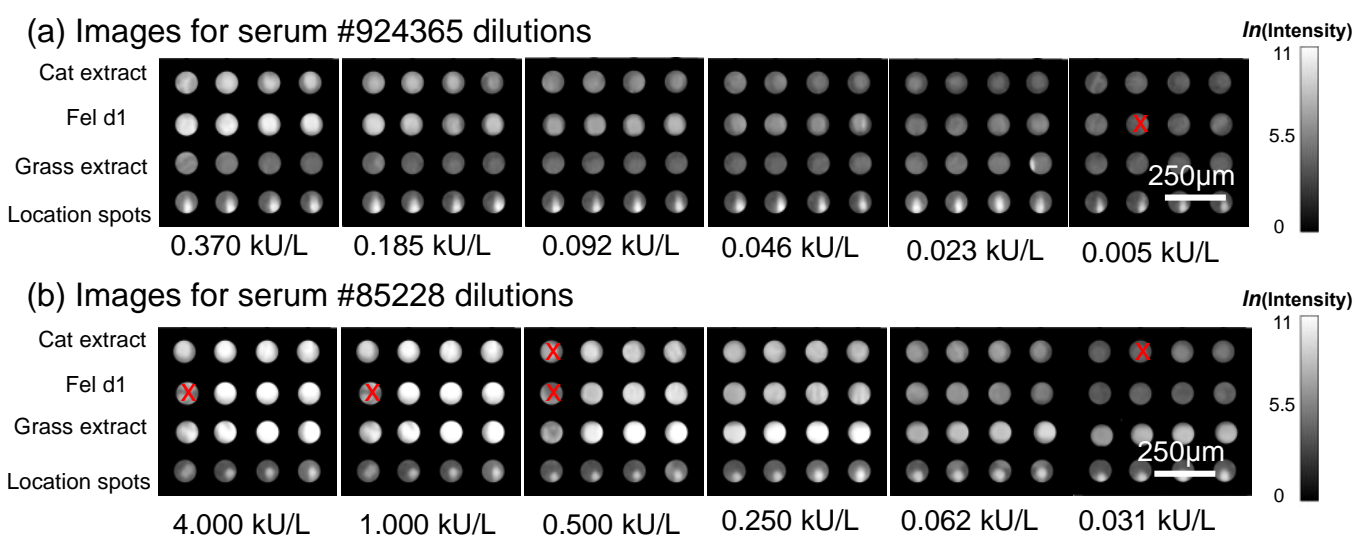

Figure 4. Fluorescent images of microspots at different $\lg E$ antibody concentrations obtained by dilution of two different serum samples. At each dilution the Feld1 spots have higher fluorescence intensities than the crude cat extract spots, confirming that purified allergen has stronger binding capacity and potentially higher diagnostic sensitivity than crude extracts. 


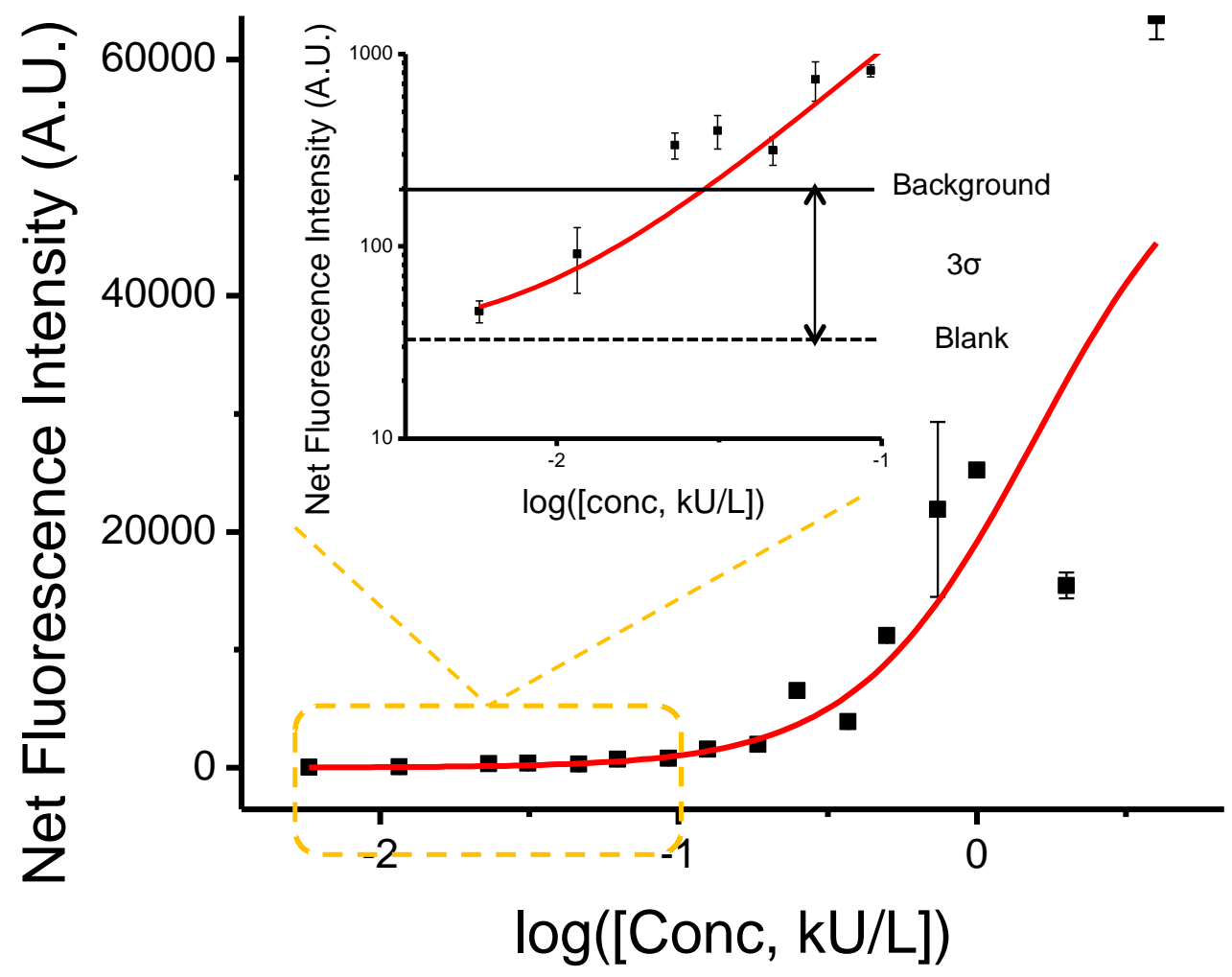

Figure 5. Standard curve for Fel d1 allergen detection. The black solid line in the inset shows the background intensity, which is the blank intensity from the negative control (indicated by the dashed line) plus three times the standard deviation. We consider fluorescence signals above the background intensity as detectable. Therefore, the lowest detectable concentration for Fel d1-specific antibody is $\sim 0.02 \mathrm{kU} / \mathrm{L}$, which is lower than that measured by the ImmunoCAP system. 


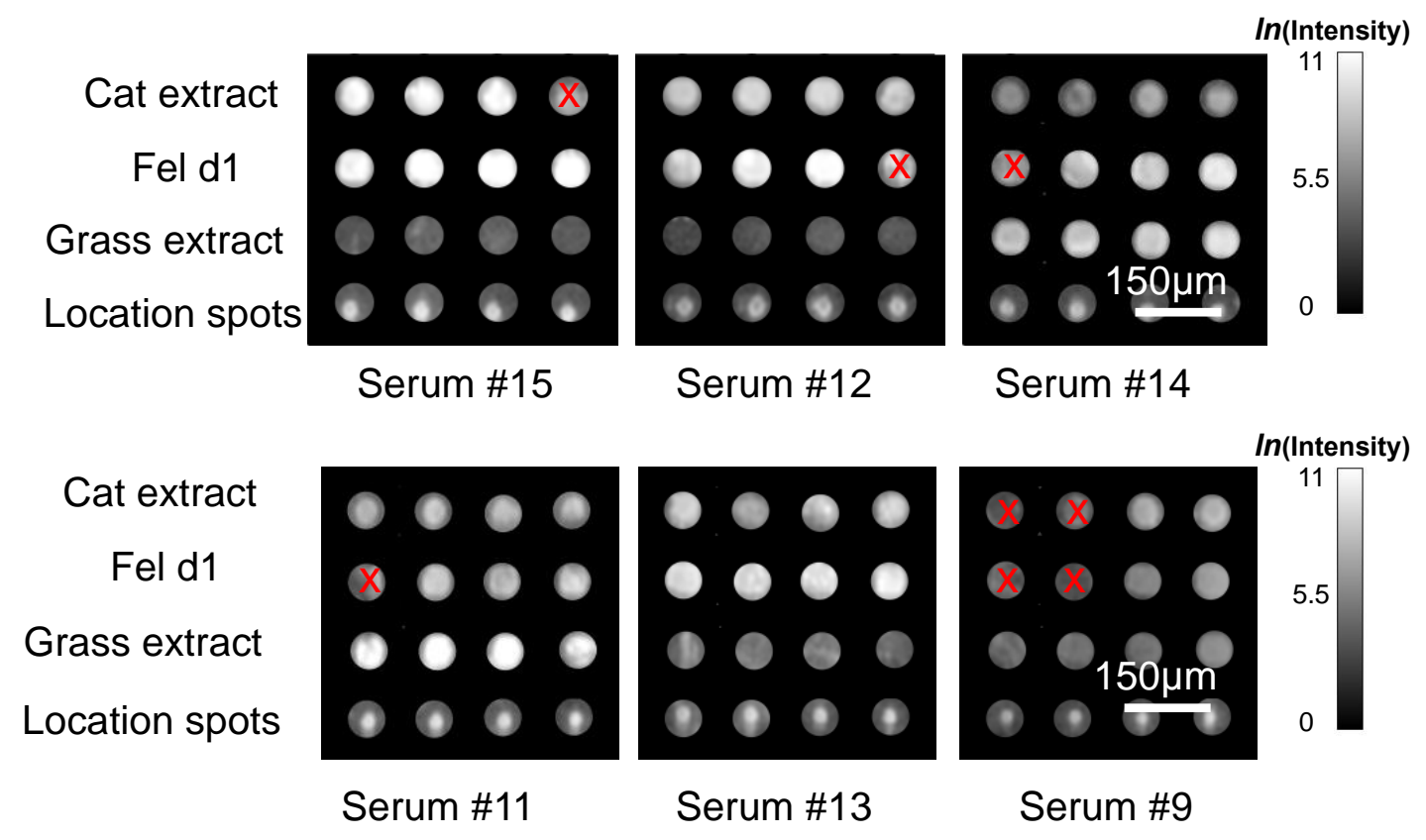

Figure 6. Representative images of the allergen spots assayed with patient sera at various $\lg E$ levels ranging from $0.089 \mathrm{kU} / \mathrm{L}$ to $40 \mathrm{kU} / \mathrm{L}$. Note that the PCEF array system is capable of detecting the low concentration of lgE in serum \#9, which is not observable using the ImmunoCAP system. 